\title{
Interventions to Reduce Neurological Symptoms in Patients with GBM Receiving Radiotherapy: From Theory to Clinical Practice
}

\author{
LUCIA PALOMBI $^{1 *}$, PAOLO MARCHETTI ${ }^{2}$, MAURIZIO SALVATI $^{3}$, \\ MATTIA FALCHETTO OSTI ${ }^{4}$, LUIGI FRATI ${ }^{3}$ and ALESSANDRO FRATI ${ }^{3}$ \\ ${ }^{1}$ Department of Neurological Sciences-Neurosurgery, Faculty of Medicine, and \\ Departments of ${ }^{2}$ Oncology, and ${ }^{4}$ Radiation Oncology, \\ Faculty of Medicine and Psychology, La Sapienza University of Rome, Rome, Italy; \\ ${ }^{3}$ IRCCS INM Neuromed, Pozzilli, Italy
}

\begin{abstract}
Background: Patients affected by glioblastoma often develop cerebral oedema as a life-threatening complication. Although there is no approved pharmacological intervention, such cerebral oedema is usually treated with dexamethasone. Dexamethasone has been shown in experimental studies to reduce cerebral oedema with only few mineralocorticoid side-effects. The goal of our study was to examine its efficacy in reducing the emergence of neurological deficits during the Stupp protocol. Patients and Methods: We studied a retrospective cohort of 459 patients, assigned in controlled groups: in group A, patients received radiochemotherapy followed by adjuvant chemotherapy; in group $B$, patients received an equivalent combined treatment with dexamethasone. Results: The frequency of neurological symptoms was significantly lower in dexamethasone-treated patients. Conclusion: Early diagnosis and prevention of cerebral oedema are important because functional consequences can be anticipated with an appropriate medical treatment. Thus, our study reveals that dexamethasone acts to prevent the appearance of neurological symptoms in patients with brain tumour.
\end{abstract}

A brain tumour occurs when abnormal cells grow within the brain (1). The most common types of primary brain tumours

\footnotetext{
*Present address: Department of Medical Oncology, Ospedale Policlinico San Martino, Genova, Italy.

Correspondence to: Lucia Palombi, Via di Casal Boccone 256, Rome, Italy. Tel: +39 3392652852, Fax: +39 063700047, e-mail: luciapalombi@gmail.com

Key Words: Dexamethasone, QoL, oedema, GBM, brain tumour, neurological symptoms.
}

in adults are gliomas and astrocytic tumours, while the most common in children are malignant medulloblastomas $(2,3)$. Glioblastoma multiforme (GBM), in particular, is the most aggressive (grade IV) and most common type of malignant brain tumour (with a median survival of 12-17 months without treatment). The overall (OS) and progression-free (PFS) survival of these patients are very poor due to the aggressiveness of the disease. Outcome can vary considerably depending on the type of tumour and how far it has spread at diagnosis (3).

The standard therapy for GBM consists of the maximal surgical resection of the tumour, followed by radiotherapy between 2 and 4 weeks after surgery, and then by chemotherapy. Minimally-invasive techniques are becoming the dominant trend in neurosurgical oncology (4). Postoperative radiotherapy and chemotherapy are integral parts of the therapeutic standard for malignant tumours (5).

Brain tumours produce symptoms that can vary depending on the part of the brain involved. Often it is also possible to record the emergence of neurological symptoms due to the 'mass effect' given by the appearance of cerebral oedema around the tumour. The increase of cerebral interstitial or intracellular fluid is related to different mechanisms and hyperosmolar solutions can be used to modify the brain bulk, preventing neurological deterioration $(6,7)$. The neurological symptoms may include headaches, seizures, vomiting, visual and mental changes $(2,8)$. More specific problems may include difficulty in walking, talking and feeling $(2,3)$. As the disease progresses, changes in personality or behaviour may also occur $(3,9,10)$.

In order to minimize neurological symptoms and treatmentrelated side-effects, patients with cancer are given dexamethasone, for its anti-inflammatory and immunosuppressant effects (11) to counteract the side-effects of antitumor treatments or it may be used to reduce oedema which 
could eventually compress other brain structures (2). Despite the lack of randomized and controlled trials, dexamethasone can reduce cerebral oedema, even if the choice of the drug, the timing of administration and the dose applied are still under strong debate (12). The most frequent side-effects can include a rise in serum glucose level, peripheral oedema, psychiatric disorders and Cushing's syndrome. Life-threatening complications (e.g. gastrointestinal ulcer or thromboembolism) remain rare, even if the side-effects of dexamethasone itself can increase over time.

The aim of our study was to evaluate whether the emergence of neurological symptoms during the course of radiation therapy for patients diagnosed with GBM could be reduced by taking dexamethasone, analysing its dosage and duration of intake.

\section{Patients and Methods}

We retrospectively analyzed a consecutive series of 459 patients diagnosed with GBM who received definitive therapy at the Department of Neurosurgery at the Umberto I Policlinico, La Sapienza University, Rome, Italy from 2002 to 2015.

The inclusion criteria were: previous neurosurgery; indication to perform radiation treatment and to end it; indication to perform chemotherapy, both combined and adjuvant; the Eastern Cooperative Oncology Group performance status (PS) 0-1; no comorbidities; one or more lesions associated with cerebral oedema.

Exclusion criteria were: patients with terminal status (PS 3) and patients requiring a minimal therapy care; previous steroid therapy in progress; tumour unresponsive to cancer therapy; lack of patient compliance in taking the prescribed medication.

The following prognostic factors were analysed: age, gender, Karnofsky performance status (KPS), tumour location and neurological onset.

The following neurological symptoms due to cerebral oedema were considered: headache, nausea, vomiting, loss of visual acuity, spatial and temporal disorientation, dizziness, numbness or weakness, seizures, incontinence, loss of coordination or balance, altered level of consciousness, aphasia or dysarthria, memory impairment, olfactory hallucinations, syndrome of intracranial hypertension and hydrocephalous (13).

All patients with primary GBM were postoperatively exposed to radiation (in daily fractions of 2 Gy given 5 days per week for 6 weeks, for a total of $60 \mathrm{~Gy}$ ) delivered with concurrent $75 \mathrm{mg} / \mathrm{m}^{2}$ temozolamide daily on days $1-7$, from the first to the last day of radiotherapy (combined radio- and chemotherapy). They were then further treated with adjuvant temozolomide at 150 to $200 \mathrm{mg} / \mathrm{m}^{2}$ days 1-5 every 28 days (Stupp protocol). An assessment of the neurological symptoms was performed every 2 weeks for 6 weeks during the combined treatment and every 28 days following the adjuvant treatment. Firstly, patients were asked to describe their symptoms and then a neuro examination was performed, including: the analysis of the mental state and of the symbolic functions (among which principally language), investigation of the cranial nerves and finally the evaluation of motor and sensory skills, balance and coordination. Patients received adjuvant therapy until one of the following occurred: progression of illness, discontinuation due to toxicity, or suspension following 12 months of radiation.
Patients were thus assigned in controlled groups, as homogeneously as possible for all the variables considered: group $A(N=249$ patients) received the Stupp protocol alone, and group $\mathrm{B}(\mathrm{N}=210$ patients) received equivalent combined treatment plus dexamethasone. Our dexamethasone-based medical therapy started with a $4 \mathrm{mg}$ daily dose of dexamethasone for 6 weeks during the combined treatment (42 doses), then patients were switched to an alternate-day regimen (dayon/day-off) for a further 14 days. After that, they were given half the dose $(2 \mathrm{mg} /$ day $)$ maintaining the alternate-day regimen for 7 more days and, finally, ended the steroid therapy. At the onset of symptoms, even patients who did not undergo medical therapy with corticosteroids, started it in association with mannitol.

The chi-square test was used for statistical analyses of the changes observed and highlight the significance of data differences. Following the standard procedure, $p$-values were considered significant if 0.05 or less.

The Ethics Committee was consulted and patients gave informed written consent to participation.

\section{Results}

A cohort of 459 patients was enrolled in the study, 281 $(61.22 \%)$ men and $178(38.77 \%)$ women, from 23 to 87 years old. As already explained, patients were divided into two groups: group A received radiochemotherapy alone (Stupp protocol) as opposed to group B who were also administered a dexamethasone-based treatment. During our study, no patients presented any of the side-effects related to the use of dexamethasone itself, hence the KPS of our patients always remained between 0 and 1 . No patient showed a deterioration of quality of life (QoL), indeed in almost all cases there was a clear improvement.

We found that 444 patients presented single lesions, while 15 patients had multiple ones. Table I summarizes the area and side of the brain affected by the target lesions observed in our sample. As is shown in Table I, the most frequent lesions were found in the temporal $(\mathrm{N}=141)$ and frontal $(\mathrm{N}=140)$ lobes, followed by the parietal $(\mathrm{N}=48)$, temporo-parietal $(\mathrm{N}=34)$, occipital $(\mathrm{N}=30)$ and fronto-temporal $(\mathrm{N}=29)$ lobes. Parietaloccipital $(\mathrm{N}=17)$ lesions, as well as fronto-parietal $(\mathrm{N}=12)$ and temporo-occipital $(\mathrm{N}=10)$ lesions, were also observed, while lesions in other brain areas were less frequently encountered (e.g. six cerebellar lesions, five lesions of the centrum semiovale, two thalamic lesions: for details refer to Table I). Furthermore, lesions were equally distributed between the right $(\mathrm{N}=236)$ and left side $(\mathrm{N}=239)$ of the brain, with only 14 lesions that were bilateral (Table I).

Table II summarizes the neurological symptoms observed in the two separate groups of patients as well as the statistical correlation between the two groups. As can be deduced from Table II, patients in group A, who did not receive dexamethasone-based treatment, were observed to suffer mostly from aphasia or dysarthria (32.93\%), sense of numbness or weakness $(25.3 \%)$, seizures $(20.88 \%)$, headaches $(20.48 \%)$ and an altered level of consciousness $(18.47 \%)$. 
Table I. Localization of target lesions in patients with glioblastoma.

\begin{tabular}{|c|c|c|c|c|c|}
\hline Localization & Right & Left & Bilateral & Total no. & $\%$ Of total \\
\hline Temporal & $59(25 \%)$ & $81(33.89 \%)$ & $1(7.14 \%)$ & 141 & 28.83 \\
\hline Frontal & $63(26.69 \%)$ & $69(28.87 \%)$ & $8(57.14 \%)$ & 140 & 28.63 \\
\hline Parietal & $31(13.14 \%)$ & $16(6.69 \%)$ & $1(7.14 \%)$ & 48 & 9.82 \\
\hline Temporo-parietal & $16(6.78 \%)$ & $18(7.53 \%)$ & 0 & 34 & 6.95 \\
\hline Occipital & $19(8.05 \%)$ & $11(4.60 \%)$ & 0 & 30 & 6.13 \\
\hline Fronto-temporal & $12(5.08 \%)$ & $16(6.69 \%)$ & $1(7.14 \%)$ & 29 & 5.93 \\
\hline Parietal-occipital & $9(3.81 \%)$ & $5(2.09 \%)$ & $3(21.43 \%)$ & 17 & 3.48 \\
\hline Fronto-parietal & $3(1.27 \%)$ & $9(3.77 \%)$ & 0 & 12 & 2.45 \\
\hline Temporo-occipital & $9(3.81 \%)$ & $1(0.42 \%)$ & 0 & 10 & 2.04 \\
\hline Cerebellar & $2(0.85 \%)$ & $4(1.67 \%)$ & 0 & 6 & 1.23 \\
\hline Centrum semiovale & $3(1.27 \%)$ & $2(0.84 \%)$ & 0 & 5 & 1.02 \\
\hline Temporo-parietal-occipital & $3(1.27 \%)$ & $1(0.42 \%)$ & 0 & 4 & 0.82 \\
\hline Corpus callosum & $3(1.27 \%)$ & $1(0.42 \%)$ & 0 & 4 & 0.82 \\
\hline Fronto-temporal-parietal & $2(0.85 \%)$ & $1(0.42 \%)$ & 0 & 3 & 0.61 \\
\hline Paratrigonal & $1(0.42 \%)$ & $1(0.42 \%)$ & 0 & 2 & 0.41 \\
\hline Thalamic & $1(0.42 \%)$ & $1(0.42 \%)$ & 0 & 2 & 0.41 \\
\hline Para-hippocampal & 0 & $1(0.42 \%)$ & 0 & 1 & 0.20 \\
\hline Petro-clival & 0 & 1 & 0 & 1 & 0.20 \\
\hline Total & 236 & 239 & 14 & 489 & 100 \\
\hline
\end{tabular}

Spatial and temporal disorientation $(11.64 \%)$, loss of visual acuity $(9.2 \%)$, loss of coordination and balance (memory impairment $(6.02 \%)$ and vomiting $(\mathrm{N}=10.4 .01 \%)$ were also observed in this group of patients. Other neurological symptoms, such as dizziness, nausea, hypertension etc., only rarely occurred (refer to group A in Table II for details).

Patients in group B, instead, appeared to be more subject to seizures $(16.19 \%)$, sense of numbness or weakness $(15.23 \%)$ and headaches $(13.3 \%)$, followed by an altered level of consciousness (aphasia or dysarthria $(8.09 \%)$ and memory impairment $(3.8 \%)$. Other neurological symptoms were negligible (refer to group B in Table II for details).

When the two groups were compared with each other, patients in group A were found to suffer significantly more than patients in group B from spatial and temporal disorientation $(p=0.0001)$, loss of coordination or balance $(p=0.0003)$ and an altered level of consciousness $(p=0.0013)$. Loss of visual acuity $(p=0.001)$, numbness or weakness $(p=0.001)$, seizures $(p=0.001)$, aphasia or dysarthria $(p=0.001)$ and headaches $(p=0.009)$ were also more significantly observed in patients of group A than group B. Interestingly, other neurological symptoms, for example nausea $(p=0.5)$, dizziness $(p=0.25)$, incontinence $(p=0.31)$ and memory impairment $(p=0.14)$ appeared to be unaffected by the treatment patients (Table II).

\section{Discussion}

The treatment of patients affected by GBM poses a challenge as the median patient survival rate after diagnosis is from 12 to 17 months with radiation and temozolomide treatment
Table II. Neurological examinations showing symptoms of patients of group A (Stupp protocol alone) compared to those of group B (Stupp protocol + dexamethasone).

\begin{tabular}{lrrl}
\hline Symptom & Group A, & Group B, $p$-Value \\
& $\mathrm{n}$ & $\mathrm{n}$ & \\
\hline Headache & 51 & 28 & 0.009 \\
Nausea & 2 & 1 & 0.5 \\
Vomiting & 10 & 2 & 0.02 \\
Loss of visual acuity & 23 & 6 & 0.001 \\
Spatial and temporal disorientation & 29 & 6 & 0.0001 \\
Dizziness & 5 & 2 & 0.25 \\
Numbness or weakness & 63 & 32 & 0.001 \\
Seizures & 52 & 34 & 0.001 \\
Incontinence & 1 & 0 & 0.31 \\
Loss of coordination or balance & 20 & 3 & 0.0003 \\
Altered level of consciousness & 46 & 20 & 0.0013 \\
Aphasia or dysarthria & 82 & 17 & 0.001 \\
Memory impairment & 15 & 8 & 0.14 \\
Olfactory hallucination & 1 & 0 & 0.31 \\
Syndrome of intracranial hypertension & 4 & 0 & 0.04 \\
Hydrocephalous & 1 & 0 & 0.31 \\
\hline
\end{tabular}

(14). Several factors play a role in the prognosis, such as age, pre-operative PS and extent of resection $(15,16)$.

Temozolomide has been shown to improve OS and PFS when given concurrently with RT in patients newly diagnosed with GBM $(14,17,18)$. Moreover, adjuvant temozolomide after completion of RT is a positive prognostic indicator of improved OS and PFS $(14,18,19)$. 
Patients with glioma face many challenges owing both to the disease and to disease-specific consequences. Therefore, increasing attention has been paid in recent years to patient QoL (20). The impact on quality of life, as well as the psychological and social aspects of the neurological alterations, must not be taken lightly, especially in young patients with a long-life expectancy. Moreover, caregivers must understand the complexity of their patients' symptom experience and its impact on their daily life.

Dexamethasone is commonly used in the management of cerebral oedema in patients diagnosed with GBM due to its anti-inflammatory properties (21). It is often prescribed at diagnosis to reduce tumour-associated vasogenic oedema; dexamethasone administration is often also continued after biopsy or resection to reduce postoperative oedema, and further continued during RT to reduce oedema associated with radiation (22). More importantly, dexamethasone is not expensive and is available in most areas of the world, making it an ideal candidate for the treatment of patients with GBM by applying a standard protocol (23).

Dexamethasone should be tapered in a manner customized to the patient and a generalized dose scheme should not be used. Side-effects, in fact, are common, and they increase in frequency and severity with increasing dose and duration of therapy. For this reason, patients should be carefully monitored for endocrine, muscular, skeletal, gastrointestinal, psychiatric, and haematological complications (22). Otherwise, in certain cases, patients have been shown to need an increase in glucocorticoids if they present focal neurological symptoms (hemiparesis, visual field deficit, or aphasia) or global symptoms (headache, nausea, or decreased appetite) (21).

In accordance with the American College of Radiology Appropriateness Criteria, there is no evidence to support the routine use of dexamethasone in patients with brain tumours in the absence of neurological signs or symptoms (24).

Our study revealed that dexamethasone had a positive effect in preventing the appearance of neurological symptoms. Most neurological symptoms were significantly less frequent in patients of group B, exposed to an additional dexamethasone-based medical therapy, than patients of group A, who had been subject to combined radiochemotherapy alone (Table II). Our results are in line with previous studies in which dexamethasone had a positive effect on all symptoms, mainly those of the respiratory tract $(25,26)$.

Furthermore, in our study we did not record any sideeffects related to dexamethasone therapy, except for sporadic detections of increased glycaemia.

Our results thus suggest that it would be good practice to treat patients with GBM with dexamethasone upfront, concurrent with RT plus temozolomide in order to prevent the neurological effects related to cerebral oedema. With this aim in mind, we are now beginning to administer dexamethasone, following the procedure described here, to all patients from the time they start RT with the hope of improving patient QoL during exposure to this medical treatment.

\section{References}

1 General Information about Adult Brain Tumours. NCI. 2014-0414. Retrieved 8 June 2014. https://www.cancer.gov/types/brain/ patient/adult-brain-treatment-pdq

2 Adult Brain Tumours Treatment. NCI. 2014-02-28. Retrieved 8 June 2014. https://www.cancer.gov/types/brain/patient/adultbrain-treatment-pdq

3 World Health Organization: World Cancer Report 2014. IARC Press, International Agency for Research on Cancer. Chapter 5: 16,2014

4 Spetzler RF and Sanai N: The quiet revolution: Retractorless surgery for complex vascular and skull base lesions. J Neurosurg 116(2): 291-300, 2012.

5 Knizhnik AV, Roos WP, Nikolova T, Quiros S, Tomaszowski KH, Christmann M and Kaina B: Survival and death strategies in glioma cells: autophagy, senescence and apoptosis triggered by a single type of temozolomide-induced DNA damage. PLoS One 8(1): e55665, 2013.

6 Bastin ME, Sinha S, Whittle IR and Wardlaw JM: Measurements of water diffusion and T1 values in peritumoural oedematous brain. Neuroreport 13: 1335-1340, 2002.

7 Dostal P, Dostalova V, Schreiberova J, Tyll T, Habalova J, Rehak S and Cesak T: A Comparison of equivolume, equiosmolar solutions of hypertonic saline and mannitol for brain relaxation in patients undergoing elective intracranial tumor surgery: a randomized clinical trial. J Neurosurg Anesthesiol 27(1): 51-56, 2015.

8 Longo DL, Fauci AS, Kasper D, Hauser SL, Jameson JL and Loscalzo J: Seizures and Epilepsy. In: Harrison's Principles of Internal Medicine. McGraw-Hill Companies, Inc. p. 3258 (18th ed.), New York, USA, 2014.

9 Lucas MR: Psychosocial implications for the patient with a highgrade glioma. J Neurosci Nurs 42(2): 104-108, 2010.

10 Sterckx W, Coolbrandt A, de Casterle BD, Van den Heede K, Decruyenaere M, Borgenon S, Mees A and Clement P: The impact of a high-grade glioma on everyday life: a systematic review from the patient's and caregiver's perspective. Eur J Oncol Nurs 17(1): 107-117, 2013.

11 Dexamethasone: The American Society of Health-System Pharmacists. Retrieved Jul 29, 2015. https://www.drugs.com/ monograph/dexamethasone.html

12 Artzi M, Bokstein F, Blumenthal DT, Aizenstein O, Liberman G, Corn BW and Ben Bashat D: Differentiation between vasogenic-edema versus tumour-infiltrative area in patients with glioblastoma during bevacizumab therapy: a longitudinal MRI study. Eur J Radiol 83(7): 1250-1256, 2014.

13 Gantner D, Moore EM and Cooper DJ: Intravenous fluids in traumatic brain injury: what's the solution? Curr Opin Crit Care 20(4): 385-389, 2014.

14 Stupp R, Mason WP, van den Bent MJ, Weller M, Fisher B, Taphoorn MJ, Belanger K, Brandes AA, Marosi C, Bogdahn U, Curschmann J, Janzer RC, Ludwin SK, Gorlia T, Allgeier A, Lacombe D, Cairncross JG, Eisenhauer E and Mirimanoff RO: Radiotherapy plus concomitant and adjuvant temozolomide for glioblastoma. N Engl J Med 352(10): 987-996, 2005. 
15 Albert FK, Forsting M, Sartor K, Adams HP and Kunze S: Early postoperative magnetic resonance imaging after resection of malignant glioma: objective evaluation of residual tumour and its influence on regrowth and prognosis. Neurosurgery 34(1): 4560, 1994.

16 Lacroix M, Abi-Said D, Fourney DR, Gokaslan ZL, Shi W, DeMonte F, Lang FF, McCutcheon IE, Hassenbusch SJ, Holland E, Hess K, Michael C, Miller D and Sawaya R: A multivariate analysis of 416 patients with glioblastoma multiforme: prognosis, extent of resection, and survival. J Neurosurg 95(2): 190-198, 2001.

17 Ronning PA, Helseth E, Meling TR and Johannesen TB: A population-based study on the effect of temozolomide in the treatment of glioblastoma multiforme. Neuro Oncol 14(9): 1178$1184,2012$.

18 Zczepanek D, Marchel A, Moskala M, Krupa M, Kunert P and Trojanowski T: Efficacy of concomitant and adjuvant temozolomide in glioblastoma treatment. A multicentre randomized study. Neurol Neurochir Pol 47(2): 101-108, 2013.

19 Stupp R, Dietrich PY, Ostermann Kraljevic S, Pica A, Maillard I, Maeder P, Meuli R, Janzer R, Pizzolato G, Miralbell R, Porchet F, Regli L, de Tribolet N, Mirimanoff RO and Leyvraz $S$ : Promising survival for patients with newly diagnosed glioblastoma multiforme treated with concomitant radiation plus temozolomide followed by adjuvant temozolomide. J Clin Oncol 20(5): 1375-1382, 2002.

20 Boele FW, van Uden-Kraan CF, Hilverda K, Reijneveld JC, Cleijne W, Klein M and Verdonck-de Leeuw IM: Attitudes and preferences toward monitoring symptoms, distress, and quality of life in glioma patients and their informal caregivers. Support Care Cancer 24(7): 3011-3022, 2016.

21 Sur P, Sribnick EA, Patel SJ, Ray SK and Banik NL: Dexamethasone decreases temozolomide-induced apoptosis in human glioblastoma T98G cells. Glia 50(2): 160-167, 2005.
22 Deutsch MB, Panageas KS, Lassman AB and DeAngelis LM: Steroid management in newly diagnosed glioblastoma. J Neurooncol 113(1): 111-116, 2013.

23 Liu G, Segrè J, Gülmezoglu AM,3 Mathai M, Smith JM, Hermida J, Simen-Kapeu A, Barker P, Jere M, Moses E, Moxon SG, Dickson KE, Lawn JE and Althabe F: Antenatal corticosteroids for management of preterm birth: a multi-country analysis of health system bottlenecks and potential solutions. BMC Pregnancy Childbirth 15(Suppl 2): S3, 2015.

24 Kostaras X, Cusano F and Easaw J: Use of dexamethasone in patients with high-grade glioma: a clinical practice guideline. Curr Oncol 21(3): 493-503, 2014.

25 Gaspar LE, Gutin PH, Rogers L, Schneider JF, Larson D, Bloomer WD, Buckley JA, Gibbs FA, Lewin AA, Loeffler JS, Malcolm AW, Mendenhall WM, Schupak KD, Shaw EG, Simpson JR, Wharam MD Jr. and Leibel S: Pre-irradiation evaluation and management of brain metastases. American College of Radiology. ACR Appropriateness Criteria. Radiology 215: 1105-1110, 2000.

26 Marwick JA, Caramori G, Casolari P, Mazzoni F, Kirkham PA, Adcock IM, Chung KF and Papi A: A role for phosphoinositol 3-kinase delta in the impairment of glucocorticoid responsiveness in patients with chronic obstructive pulmonary disease. J Allergy Clin Immunol 125(5): 1146-1153, 2010.

Received January 29, 2018

Revised February 22, 2018

Accepted February 27, 2018 